\title{
DERECHOS FUNDAMENTALES Y PROCEDIMIENTO EN LA OBRA DE PETER HÄBERLE*
}

\author{
ANGELO SCHILLACI ${ }^{* *}$
}

\begin{abstract}
RESUMEN: En el artículo, el Autor destaca la aportación de Peter Häberle a la dogmática de los Derechos Fundamentales, con referencia a su obra "Grundrechte im Leistungsstaat" y a la relación entre Derechos fundamentales y procedimientos públicos de decisión y prestación. La relación entre derechos fondamentales y procedimento afecta nudos centrales en la dogmática de los derechos. En particular, el análisis de esta relación permite individuar instrumentos más adecuados de protección de los derechos, así como posibilidades de realización de los derechos a través de la participación. Al mismo tiempo, eso conlleva una reflexión acerca de la influencia de los derechos en el modelo de legitimación del Estado constitucional, poniendo en relieve - a través del procedimiento - la relación entre libertad, participación y cooperación en el ejercicio del poder público en aras a elecciones enmarcadas en el cuadro constitucional.
\end{abstract}

PALABRAS CLAVE: Derechos Fundamentales; Procedimiento; Peter Häberle; Participación.

\begin{abstract}
In the article, the Author works on the contribution of Peter Häberle in the studies on Fundamental Rights, especially for what concerns his article "Grundrechte im Leistungsstaat" and the relation between Fundamental Rights and public procedures of decision. The relation between fundamental rights and public procedures affects crucial problems in the studies on fundamental rights. In particular, the analysis of this relation could lead to the individuation of more adequate instruments of protection of fundamental rights. At the same time, it stimulates a deeper reflection on the influence of fundamental rights on the legitimity of constitutional state, recalling, in particular, the relation between freedom, participation and procedure in order to choices inspired by the constitution.
\end{abstract} KEYWORDS: Fundamental Rights; Procedure; Peter Häberle; Participation.

RESUMO: No artigo, o autor destaca o aporte de Peter Häberle à dogmática dos direitos fundamentais, com referência à sua obra "Grundrechte im Leistungsstaat (Direitos fundamentais no Estado prestacional)" e à relação entre direitos fundamentais e procedimentos públicos de decisão e prestação. A relação entre direitos fundamentais e procedimento afeta nódulos centrais na dogmática dos direitos. Em particular, a análise desta relação permite identificar instrumentos mais adequados para proteção dos direitos, assim como possibilidades de realização dos direitos através da participação. Ao mesmo tempo, isso resulta em uma reflexão acerca da influência dos direitos no modelo de legitimação do Estado constitucional, pondo em evidência - através do procedimento - a relação entre liberdade, participação e cooperação no exercício do poder público conforme opções determinadas no quadro constitucional.

PALAVRAS-CHAVE: Direitos Fundamentais; Procedimento; Peter Häberle; Participação.

Artigo recebido em 08.06.2010. Artigo aceito para publicação mediante convite.

* Professor Doutor da Universidade La Sapienza, Roma. 
SUMARIO: 1. Derechos y procedimento entre dimensión individual e institucional; 2. Participación en el procedimiento y procesos de integración; 3. El status activus processualis; Bibliografia.

SUMMARY: 1. Rights and procedure between the individual and the institutional dimension; 2. Participation in the procedure and in integration processes; 3 . The status activus processualis; Bibliography.

SUMÁRIO: 1. Direitos e procedimento entre dimensão individual e institucional; 2. Participação no procedimento e processos de integração; 3. O status activus processualis; Bibliografia.

1. La relación entre derechos fondamentales y procedimento afecta nudos centrales en la dogmática de los derechos. En particular, el análisis de esta relación nos permite individuar instrumentos más adecuados de protección de los derechos, así como posibilidades de realización de los derechos a través de la participación. Al mismo tiempo, el estudio de esa relación conlleva una reflexión acerca de la influencia de los derechos en el modelo de legitimación del Estado constitucional, poniendo en relieve - a través del procedimiento - la relación entre libertad, participación y cooperación en el ejercicio del poder público en aras a elecciones enmarcadas en el cuadro constitucional.

Desde esta perspectiva, puede ser particularmente útil recuperar la reflexión de Peter Häberle, en el conocido ensayo de 1972 sobre «los derechos fundamentales en el Estado prestacional", en el que el autor expone una resistematización de la concepción y de la comprensión dogmática de los derechos fundamentales en relación con los múltiples y nuevos desafios del Estado social. Frente a un Estado que progresivamente se hace responsable de la creación de las condiciones materiales y espirituales que hagan posible el disfrute pleno de los derechos fundamentales, la cumplida autodeterminación de la persona - piedra angular del sistema constitucional - impone la reconsideración del sistema de las libertades fundamentales, reconociendo plena relevancia a la dimensión positiva de su ejercicio responsable.

El paso de una concepción "negativa" a una concepción "positiva" de la libertad ${ }^{2}$ se acompaña, en el Estado prestacional, de una valoración positiva del vínculo existente entre ejercicio responsable de los derechos y participación en los procesos de decisión pública, a través de su sujección a esquemas procedimentales. Al mismo tiempo, la estratificación de significados (Bedeutungsschichten) de los derechos fundamentales - la progresiva profundización de su multi-dimensionalidad, como espejo de una «Menschenbild», consciente de la complejidad de las históricas

${ }^{1}$ P. HÄBERLE, Grundrechte im Leistungsstaat, en VER. DER DEUTSCHEN STAATSRECHTSLEHRER, Grundrechte im Leistungsstaat/Die Dogmatik der Verwaltungsrechts vor den Gegenwarts Aufgaben der Verwaltung, Berlin-New York, De Gruyter 1972, pp. 43 ss., trad. it. I diritti fondamentali nello Stato delle prestazioni, en P. HÄBERLE, Cultura dei diritti e diritti della cultura nello spazio costituzionale europeo, Milán, Giuffré, 2003, pp. 133 ss.

${ }^{2}$ Sobre este pasaje, v. por todos P. RIDOLA, Diritti fondamentali. Un'introduzione, Turín, Giappichelli 2006, especialmente pp. 148 ss., también, - en la óptica específica del presente trabajo - H. GOERLICH, Grundrechte als Verfahrensgarantien, Baden Baden, Nomos 1981, especialmente pp. 176 ss., 190-191. 
condiciones de vida de los miembros de la comunidad ciudadana - nos abre a una profunda remeditación del mismo modelo de legitimación del Estado constitucional ${ }^{3}$. Desde una concepción de la libertad como límite externo al ejercicio del poder público, se pasa progresivamente a una concepción de los derechos fundamentales plenamente consciente de su capacidad de envolverse entre los procesos de integración orientados por el marco constitucional. Ha sido mérito de la concepción de Häberle - deudora de opiniones maduradas en la experiencia weimariana ${ }^{4}-1 a$ explicación de la concreta modalidad de articulación de la relación entre derechos y procesos de integración, aclarando la relevancia central de la participación en módulos procedimentales como fundamento de la construcción de procesos de autodeterminación y autorealización del particular, con repercusiones directas sobre la propia legitimación del proceso político a través de la Constitución.

El vínculo entre la participación en el procedimiento y la autodeterminación a través del ejercicio de los derechos se inserta con dificultad en las líneas del pensamiento alemán sobre los derechos. Tanto desde la integración del carácter de «Abwehr» a través de la referencia a la determinación normativa de condiciones procedimentales de ejercicio de derechos, cuanto desde su relación con la actuación del derecho «in processu», de forma más general, y por lo tanto, con la tensión no resuelta entre la dimensión subjetiva y la dimensión institucional, se ponen en juego articulaciones fundamentales de la dogmática de los «Grundrechte», que sólo en parte pueden reconducirse a la bifurcación entre la concepción "liberal" y la concepción "social" de los derechos. Entorno a la pregunta fundamental sobre la relación entre derechos y participación - la realización de los derechos «a través del derecho objetivo», ¿representa una oportunidad de profundización en las condiciones de libertad del particular en la multiplicidad de las relaciones sociales de las que es protagonista? o ¿se expone el particular irremediablemente al riesgo de una deriva institucional que terminaría por absorver al individuo en dinámicas de carácter corporativo restringiendo drásticamente sus espacios de autonomía? - surgen, en definitiva, cuestiones centrales en el desarrollo histórico de la propia concepción alemana de las relaciones entre autoridad y libertad ${ }^{5}$.

\footnotetext{
${ }^{3}$ Además de P. RIDOLA, Diritti fondamentali..., cit., pp. 93 ss., fundamental sobre este punto K. HESSE, Grundzüge des Verfassungsrechts der Bundesrepublik Deutschland, 20. Aufl., Heidelberg, C. F. Müller 1999, pp. 125 ss., 127, así como ID. Bestand und Bedeutung der Grundrechte in der Bundesrepublik Deutschland, in EuGrZ 1978, pp. 427 ss., especialmente 430 ss., y Bedeutung der Grundrechte, en BENDA/MAIHOFER/VOGEL, Handbuch des Verfassungsrechts der Bundesrepublik Deutschland, Berlin, De Gruyter 1994, pp. 127 ss.

${ }^{4}$ Si bien no puede minusvalorarse la influencia de las teorizaciones smendianas, también a través de la mediación de Konrad Hesse, es particularmente significativa la mención repetida, en las primeras páginas del ensayo, a la concepción de la teoría del Estado como ciencia de la realidad, propuesta por Hermann Heller (cfr. le pp. 134 ss. tr. it.)

5 Según Paolo Ridola, Diritti fondamentali..., cit., p. 183, "tensione dell'individuo verso la propria autorealizzazione e impossibilità di sfuggire ad un quadro sociale di riferimento segnano [...] l'intera vicenda storica dei diritti costituzionali. Sobre el Doppelcharakter der Grundrechte (entendidos como derechos subjetivos de un lado, y como elementos de un ordenamiento objetivo, por el otro), cfr. HESSE, Grundzüge..., cit., pp. 127 ss. Retomando el desarrollo histórico del constitucionalismo alemán, y en particular la peculiar relevancia - verdaderamente problemática - de la dimensión comunitaria, Paolo Ridola subraya a propósito del riesgo de una completa deriva institucional - que la garantías de instituto "costituiscono
} 
El descubrimiento de un "componente de derecho procesal" (verfahrensmäßige Komponente der Grundrechte) asociado, por un lado, al carácter de defensa y, por el otro, al "contenido material" del derecho fundamental, no debe traducirse en una rígida separación entre las diversas comprensiones de los derechos fundamentales. La consciencia de la multidimensionalidad de los derechos está de hecho estrechamente ligada en Häberle (aunque no sólo, sino también por poner un ejemplo en K. Hesse y P. Ridola), a la plena consciencia de la interdependencia entre derechos y experiencia histórica, es decir, a la consciencia de la correspondencia entre dogmática de los derechos fundamentales y la "imagen de la persona humana" acogida por la Constitución: como tal, ésta tiene bien presente las dinámicas de relación y enriquecimiento recíproco entre las diversas dimensiones de los derechos. En otras palabras, la afirmación de la multidimensionalidad de los derechos debe traducirse en el enriquecimiento del contenido del derecho, y no en la exclusión de tales dimensiones en favor de otras: las diversas comprensiones de los derechos contribuyen más bien, al interactuar, a la profundización de la propia relación entre derechos y sistema constitucional.

Justamente desde esta óptica, no parece que pueda ser exacerbada - respecto a la relación entre derecho y procedimiento - la contraposición entre dimensión subjetiva y dimensión institucional. Una lectura del ensayo de 1972 que atienda, por un lado, al debate alemán de aquellos años, y por el otro al sucesivo desarrollo de la reflexión del autor, saca a la luz que es precisamente en las relaciones entre derecho y procedimiento, si bien correctamente combinadas con una concepción multidimensional de los derechos orientada por el marco constitucional, donde radica la posibilidad de superación del conflicto.

Con el ensayo de 1972, Häberle contribuye a aclarar el alcance de la recuperación de la concepción institucional de los derechos fundamentales - formulada a partir del trabajo de 1969 sobre la garantía del contenido esencial en la Ley fundamental protegiéndola frente al riesgo de desatender la dimensión de libertad individual del derecho a favor de la dimensión institucional. La ambigüedad inherente al ligamen entre garantías institucionales y declinación de la libertad positiva, como ámbito de libertad ordenado por la intervención del derecho objetivo, se disuelve en la medida en que la disciplina de las condiciones organizativas del ejercicio del derecho se ancla en formas procedimentales, abiertas a la participación del titular del derecho, y orientadas, como se verá más adelante, a la actuación de objetivos constitucionales. De este modo la reflexión de Häberle - como subraya Stern - se caracteriza por una estructura argumentativa sensible a la libertad (freiheitsfreundlich) fundada sobre la idea de un reforzamiento mutuo entre las dos dimensiones de los derechos fundamentales ${ }^{6}$.

una forma specifica di garanzia costituzionale che, pur difendendo da attacchi del legislatore «ambiti organizzatori» trova comunque giustificazione [...] nella protezione di una sfera soggettiva" (cfr. P. RIDOLA, Garanzie costituzionali e dimensioni dei diritti di libertà, en ID. Diritti di libertà e costituzionalismo, Turín, Giappichelli 1997, pp. 1 ss., 13, pero v. también ID. Diritti fondamentali..., cit., pp. 77 ss. y 89 ss.). En el mismo sentido, K. STERN, Das Staatsrecht der Bundesrepublik Deutschland, Bd. III/1. Allgemeine Lehren der Grundrechte, München, Beck, 1988, pp. 792 ss., subraya la estrecha complementariedad entre dimensión subjetiva y dimensión institucional de los derechos fundamentales, por ejemplo, en la doctrina weimariana, en el sentido de un reforzamiento de la primera a través de la segunda.

${ }^{6}$ En este sentido K. STERN, Das Staatsrecht ..., cit., p. 794. 
Si en la dimensión procedimental de los derechos fundamentales radica una pretensión de participación en los procesos públicos de decisión, la comprensión de los derechos en sentido institucional no comporta necesariamente una concepción de la libertad como "libertad ordenada", sino que revela más bien que la organización de la libertad - ya en Heller vinculada, como consecuencia de la aproximación metodológica como ciencia de la realidad, a módulos positivos de acción ${ }^{7}$ — se traduce en una realización de la libertad misma a través de la participación y la colaboración entre poderes privados y públicos: en este sentido, Häberle puede reproponer la afirmación helleriana según la cual planificación legislativa y libertad no se contradicen, porque "en la realidad social la libertad debe estar siempre organizada" ("in gesellschaftlichen Wirklichkeit die Freiheit immer organisierte werden müsse" $"$ ).

Si bien es cierto que en Häberle el Estado prestacional aparece estrechamente ligado a una concepción de la relación entre el Estado, los ciudadanos y los grupos, que restringe - aparentemente al menos - la esfera de la autonomía social como ámbito de libertad negativa, no puede olvidarse que una mutación similar en la comprensión de la relación entre esfera pública y privada se inserta en un contexto histórico caracterizado por la intervención férrea del Estado en la idea privada, en vista de la plena realización de una libertad efectiva y real. Frente a similares intrusiones y a la consciencia de que las prestaciones estatales pueden estar pueden implicar limitaciones de derechos - se hace necesaria, según Häberle, una superación del «status passivus subjectionis» como «status» fundamental mediante el replanteamiento de la relación entre Estado y ciudadano desde una base cooperativa y comunicativa ${ }^{9}$.

En este sentido, la fundación constitucional del Estado prestacional persigue - a través del descubrimiento de la dimensión procedimental de los derechos fundamentales como garantía de participación - el objetivo de que el Estado adquiera una "estructura

\footnotetext{
${ }^{7}$ Cfr. Por ejemplo el importante ensayo Democrazia politica ed omogeneità sociale (1928), en H. HELLER, Stato di diritto o dittatura?, U. Pomarici (ed.), Nápoles, ESI, 1998, pp. 9 ss.

${ }^{8}$ Grundrechte im Leistungsstaat, cit., p. 91.

${ }^{9}$ Particularmente significativo, a este propósito un fragmento (p. 59 de la versión original) en el que Häberle tras haber afirmado que el Estado prestacional se caracteriza por una nueva forma de relación externa [(Außen) -Verhältnis] con los ciudadanos y los grupos, es decir, con la sociedad - con la importante precisación de que ésta, a causa de ello, ya no es "autónoma" - sostiene que dicha relación se articula mediante la comunicación y la cooperación, en vez que a través de la mera sujeción (y ello a pesar de que en las prestaciones puedan radicar limitaciones de los derechos). Si en la referencia a la falta de autonomía de la esfera social podría radicar el riesgo de una deriva autoritaria de la concepción institucional de los derechos fundamentales, o mejor aun, de la concepción de la relación entre privado y público sometida a algunas conjugaciones de la misma (piénsese como primera aproximación a la posición de Schmitt) la fuerte acentuación de los elementos de la cooperación y del diálogo alejan tal peligro y muestra que ausencia de autonomía significa en este caso "ausencia de separación", "ausencia de indiferencia recíproca" más que "sujeción". Sobre estas bases se puede comprender la importante afirmación, de la página 59, según la cual, "el Estado prestacional se abre e integra, poco a poco, distintos ámbitos parciales de la sociedad, predisponiendo procedimientos de prestación funcionales al ejercicio y a la actuación de la libertad, pero que no están liberados de la intervención del Estado, en los que los interesados se encuentran plenamente envueltos" (trad. nuestra: cfr. el texto original: "der Leistungsstaat öffnet sich und beginnt Teilbereiche der Gesellschaft zu integrieren. Er schafft freiheitliche, aber nicht staatsftreie, Leistungsverfahren, in welchem die Betroffenen „einbezogen“ werden“).
} 
integradora", abriéndolo al ciudadano y a los grupos ${ }^{10}$. Ésto llega a afectar la propia función de los derechos fundamentales como factores de integración, descubierta y afirmada en su tiempo por Rudolf Smend.

La dimensión procedimental de los derechos reenvía así, mediante la pretensión participativa, a una "personale Verankerung" (anclaje en la persona, Goerlich) de las condiciones jurídicas de actuación y realización de los derechos fundamentales mediante el derecho objetivo; de lo que sigue que la concepción institucional se abre a las dinámicas de la integración social, superando una ordenación estática de las garantías institucionales como pilares inmóviles del edificio constitucional.

Se podría afirmar - simplificando - que la posición de Häberle supone un "encuentro" entre las elaboraciones teóricas de Smend y Heller: el ejercicio de los derechos, también a través de la participación en procedimientos de decisión pública, parece ser funcional a la realización progresiva de la homogeneidad social a través de procesos de integración profundamente condicionados por la efectividad de los propios derechos. Una conclusión similar revela el nexo de continuidad entre la reflexión de Häberle y la experiencia weimariana, especialmente allí donde se retiene que la reflexión madurada en aquel contexto sobre las garantías institucionales mirase aun reforzamiento de los tradicionales derechos de libertad y no a la pérdida de sus condiciones de derechos subjetivos por el trámite de un "encuadramiento" en el derecho objetivo.

2. Los miedos a una deriva institucional se esfuman si se considera que la propuesta de Häberle sobre la relación entre derechos fundamentales y participación en el procedimiento debe encuadrarse en el contexto de la Ley fundamental alemana, que influye de forma particularmente significativa sobre la posibilidad de atemperar las diferencias entre dimensión subjetiva y dimensión institucional. Precisamente desde las disposiciones que señalan en profundidad la articulación de la misma relación entre libertad y autoridad en el sistema constitucional alemán, - especialmente la garantía de la dignidad humana y su intangibilidad, en el art. 1 -, es posible empezar a resolver la tensión radicada en el "doble carácter" de los derechos.

Al mismo tiempo, asume una particular relevancia el encuadramiento de los procedimientos de decisión pública en el marco diseñado por la Ley fundamental, con referencia tanto a su funcionalidad a la persecución de objetivos constitucionales, tanto a la necesidad de disciplinarlos de manera conforme a los derechos. La relación entre derechos y procedimiento, por lo tanto, debe ser analizada y comprendida en el ámbito del espacio público determinado en la Constitución, es decir, en el cuadro de los procesos de integración por ella orientados ${ }^{11}$.

La participación del titular del derecho fundamental en los procedimientos de decisión pública que influyen sobre el contenido de dicho derecho se convierten, desde esta óptica, en instrumento de apertura de la propia Constitución - y de su

\footnotetext{
${ }^{10}$ Cfr. p. 149 de la versión italiana.

${ }^{11}$ Sobre le tema v. P. RIDOLA, Prime riflessioni sullo "spazio pubblico" nelle democrazie pluralistiche, en prensa en curso de publicación en el Liber amicorum per Federico Spantigati y en P. RidOLA, Diritto comparato e diritto costituzionale europeo, Torino, Giappichelli, 2010.
} 
modelo de legitimación- a la sociedad, elemento cualificante de un concepto de Constitución orientado a la realidad social ("auf die Gesamtgesellschaft und ihre Wirklichkeit ausgerichtete Verfassungsbegriff') y caracterizado en sentido "normativo-procedimental",

La dimensión institucional de los derechos fundamentales — una vez anclada en los procedimientos de decisión pública abiertos a la intervención de los titulares de los propios derechos en el marco de una participación orientada hacia objetivos constitucionales - se resuelve siempre en formas de ejercicio del derecho cada vez más sensibles al vínculo entre titular individual del derecho y comunidad política (Gemeinschaftsbezogenheit der Grundrechte). A través de la acentuación del dato de la participación como forma de ejercicio del derecho la dimensión institucional se libera de la relación asfixiante entre un sistema de valores entendido de manera estática (Wertbezogenheit der Grundrechte) y se abre progresivamente a reconstucciones más atentas a la historicidad de las garantías de instituto ${ }^{13}$. Por este cauce, se puede llegar a interpretar los derechos como elementos fundacionales de un orden objetivo ((Elemente einer objektiver Ordnung), profundamente condicionado en sentido histórico ${ }^{14}$. Gracias a este trámite, como ya hemos dicho, el ligamen entre ejercicio de los derechos fundamentales y procesos de decisión pública se convierte en un instrumento dinámico al servicio de integraciones orientadas por la Constitución.

El desarrollo de aquella intuición "smendiana" que refiere el proceso de integración de la comunidad política a la Constitución - en el sentido del anclaje de éste no sólo en los procedimientos y en las estructuras organizativas de la forma de gobierno, sino también a través de los derechos, en los contenidos materiales y en las orientaciones axiológicas expresivas de la propia Constitución - abre así nuevos espacios en aras a la superación de los riesgos de una deriva institucional de los derechos, en particular a través de la confluencia entre el ejercicio del derecho y la participación cooperativa y responsable en procedimientos de decisión pública al mismo tiempo «constitucionalmente orientados y abiertos». La progresiva "superación de la separación liberal entre la esfera civil y la esfera política" - alcanzada también en virtud de la concepción de la Constitución como sede de procesos de integración - parece alejarse del tan temido riesgo de una "confluencia de todo lo social en el ámbito de la estatalidad" y de la completa "absorción de la conflictualidad social en la organicidad de la esfera pública" ${ }^{\text {. }}$. Más bien, cuanto más se favorece la apertura de la Constitución

${ }^{12}$ Cfr. las pp. 56-57 de la versión alemana, 149 de la traducción italiana: puede subrayarse que la lectura del ensayo de 1972 a la luz de similares afirmaciones sobre la apertura de la Constitución a través del procedimiento revela perspectivas de continuidad - en parte inéditas - con las sucesivas producciones del Autor.

${ }^{13}$ Lo cual se hace particularmente evidente precisamente en la evolución del itinerario científico de Häberle, en referencia tanto al pasaje del trabajo de 1969 al ensayo de 1972 cuanto, sobretodo, al desarrollo sucesivo en materia de relaciones entre Constitución como proceso público y organización del pluralismo. Sobre el itinerario científico de Peter Häberle v. P. RidolA, Laudatio de Peter Häberle, en Rev. Der. Const. Eur., $\mathrm{n}^{\circ}$ 1/2009, pp. 389 ss.; A. A. CERVATI, Il diritto costituzionale e la comparazione nel pensiero di Peter Häberle, en ID. Per uno studio comparativo del diritto costituzionale, Turín, Giappichelli 2009, pp. 140 ss.; M. AZPITARTE SÁNCHEZ, Apuntes sobre el pensamiento de Peter Häberle en el contexto de la dogmática alemana, en Rev. Fac. Der. Univ. Granada n. 6/2003, pp. 345 ss.

${ }^{14}$ Para este pasaje, fundamental la obra de K. HeSSE, Bestand und Bedeutung..., cit., pp. 431-432 y 438.

${ }^{15}$ En este sentido, P. RIDOLA, Democrazia pluralistica e libertà associative, Milán, Giuffré 1987, pp. 2-3. 
al pluralismo social, cuanto más acoge la Constitución al pluralismo, orientándose las dinámicas a través del establecimiento de condiciones y estructuras idóneas al favorecimiento de la confrontación de los intereses, absorbiendo las tensiones más marcadas, tanto más la participación en los procesos de decisión parece integrarse en un espacio público que se convierte, por vía de la Constitución, en horizonte de la liberación de la persona y de solidaria integración social y política a través de la cooperación responsable en el ejercicio de los derechos fundamentales.

Por un lado, por lo tanto, la concepción institucional de los derechos fundamentales puede ser leída como el momento de un más amplio recorrido que conduce de una concepción de los derechos basada en la oposicion entre el particular y el Estado que se podría afirmar como formal y abstracta - a una concepción material de los mismos, es decir, anclada en la realidad de procesos de autodeterminación moral «y»" social promovidos y garantizados por la Constitución. Por otro lado, su integración a través de la dimensión procesal del ejercicio de los derechos señala un paso ulterior hacia adelante que permite disolver, mediante el reconocimiento de la relevancia de la participación, la ambigüedad propia de la tensión (dialéctica) entre dimensión «subjektiv-» y «objektiv-rechtlich» de los derechos fundamentales.

En otras palabras, la conformación de la organización administrativa y de los procedimientos de decisión pública en un sentido conforme a los derecho fundamentales ${ }^{16}$ implica directamente la apertura de estos últimos a la participación de los titulares de los derechos, y eventulamente de las organizaciones exponenciales de sus intereses.

A la luz de la Ley Fundamental de 1949 se alcanza la consciencia de que la relación inescindible entre actuación del contenido material de los derechos y procedimiento deriva directamente de la exigencia de estructuración de las garantías de instituto y su actuación a través del derecho objetivo de manera conforme a los derechos fundamentales, entendidos aun como derechos individuales y posiciones de defensa respecto a ingerencias del poder público ${ }^{17}$. La participación en el procedimiento - especialmente allí donde éste tenga como finalidad la adopción de una decisión destinada a la limitación del derecho - representa así una nueva forma expresiva de la clásica función defensiva adscrita a los derechos fundamentales. Como afirma el mismo Häberle, en este sentido, es decir reconociendo la centralidad de la participación, el momento defensivo puede retenerse completado a través del procedimiento $^{18}$.

${ }^{16}$ CFR. E. DENNINGER, Staatliche Hilfe zur Grundrechtsausübung durch Verfahren, Organisation und Finanzierung, en J. ISENSEE- P. KIRCHHOF (hrsg.), Handbuch des Staatsrechts, Heidelberg, C. Müller, 1992, 297, donde afirma que la conformación de la organización y los procedimientos debe ser "grundrechtsdienlich".

${ }^{17}$ En este sentido, v. sobretodo H. GOERLICH, Grundrechte..., cit., p. 194 ss., 195, que subraya la necesidad de articular la dimensión institucional de los derechos fundamentales mediante una estructuración a través de garantías subjetivas de participación en los procedimientos. Esto facilitaría - siempre según Goerlich el abandono de fórmulas controvertidas, como aquella - ya citada - según la cual los derechos fundamentales constituirían los elementos de un ordenamiento objetivo o aun aquella que individualiza efectos de los derechos funcionalizados a la organización administrativa. (p. 215).

${ }^{18}$ Cfr. P. HÄBERLE, Grundrechte im Leistungsstaat, cit., pp. 88-89; E. DENNINGER, Staatliche Hilfe..., cit., pp. 292-295. 
La idea de una coordinación entre libertad positiva y libertad negativa ${ }^{19}$ encuentra su propia desembocadura en las posibilidades de participación en el procedimiento entendido como instrumento de ejercicio cooperativo de los derechos fundamentales (kooperative Grundrechtsausübung) ${ }^{20}$. La referencia a la cooperación, por otro lado, no se agota en la reestructuración de la relación entre el particular y la administración pública, sino que declina de forma comunicativa la misma relación entre el individuo y la colectividad. En otras palabras, el ejercicio cooperativo de los derechos fundamentales se refiere tanto a módulos de diálogo y cooperación entre el particular y la administración pública, como al encuentro - en el procedimento - entre el particular y otros titulares del mismo o de otros derechos fundamentales, con conclusiones significativas en orden a la recomposición de los conflictos interiores al tejido pluralista ${ }^{21}$. Libertad y «participación pluralista»-podemos afirmar con Häberle - "siguen el mismo paso en tanto que los derechos de prestación no se transformen en deberes y no conlleven obligaciones que a causa, por ejemplo, de una insuficiente información o por falta de comprensión de un contexto complejo, exijan demasiado de los que tienen derecho a la participación, transformándola así en una farsa",22.

Sólo en la interacción positiva entre objetivos constitucionales y participación orientada a la efectividad de los derechos, la dimensión procesal de los derechos fundamentales en el Estado de las prestaciones puede referirse a un horizonte de liberación de la persona. Y sólo en este sentido, la dimensión procesal del ejercicio de los derechos fundamentales puede realizar una eficaz subjetivación de la dimensión institucional de los propios derechos, de su anclaje personal (personale Verankerung, Goerlich), y finalmente de la posición del sujeto (Subjektsstellung) en el sistema en conformidad con el objetivo constitucional de protección y «promoción» de la dignidad humana. La "relación comunicativa" entre ciudadano y colectividad —entendida bien como "integración", o bien como "realización del ser humano a través de los demás" - presupone como "punto de partida negativo" la "protección de la vida personal y de la libertad espiritual como libertad de alienación (Freiheit der Entäußerung)",23, confirmando así que, como afirma Ridola, la "comprensión de la garantía de los

\footnotetext{
${ }^{19}$ V. Sobre el tema, H. GOERLICH, Grundrechte..., cit., pp. 176 ss., pp. 190-191, pero también más en general, P. RIDOLA, I diritti fondamentali..., cit., pp. 148 ss.

${ }^{20}$ Cfr. E. DENNINGER, Staatliche Hilfe..., cit., p. 308.

${ }^{21}$ Sobre el tema, fundamental, Ridola, v. también Garanzie costituzionali..., cit. pp. 19 ss., especialmente para la afirmación según la cual las "inedite possibilità di sviluppo" de los derechos fundamentales en el Estado de las prestaciones "risultano condizionate anche dall'attivazione di canali di cooperazione e comunicazione fra pubblico e privato, o meglio dall'inserimento dei privati in "procedure di prestazione» nell'ambito delle quali i privati e i gruppi sociali operano come Sozialpartner degli apparati pubblici. La tenuta e l'espansione dei diritti fondamentali nello Stato sociale dipende dunque dall'interdipendenza fra prestazioni pubbliche e «capacità di prestazione» della società la cui tensione dialettica può trovare composizione attraverso procedimenti" (p. 21), entendidos como procesos de comunicación pública que "consentono il confronto tra diverse esperienze sociali". En el mismo sentido, fundamental K. HESSE, Grundzüge..., cit., pp. 160-161, pero también ID., Bestand und Bedeutung..., cit., pp. 437-438, así como ID. Bedeutung der Grundrechte, cit., p. 148.

${ }^{22}$ Así P. HÄBERLE, I diritti fondamentali..., cit., pp. 182-183.

${ }^{23}$ Para esta idea, cfr. H. GOERLICH, Grundrechte..., cit., pp. 192-193.
} 
derechos es inseparable de la premisa de que la Constitución busca el punto de equilibrio entre libertad e integración" ${ }^{24}$.

La centralidad del ser humano y la garantía de la intangibilidad de la dignidad humana no agotan sus efectos en el reconocimiento constitucional de una serie más o menos amplia de los derechos fundamentales: más bien - como se muestra en la experiencia italiana la relación entre el art. 2 y el art. 3 de la Constitución ${ }^{25}-$ reconocimiento y protección deben abrirse necesariamente a la previsión de toda una serie de prestaciones - también organizativas y procedimentales - idóneas a la promoción de la dignidad humana a través de la participación en el procedimiento entendida como forma de ejercicio consciente de los derechos fundamentales.

La citada «Subjektstellung» - posición del sujeto, comprendida como fundación del ordenamiento objetivo sobre la precedencia de la persona - no puede limitarse a proclamaciones de principio: ésta sitúa sobre la Constitución y los poderes públicos legitimados y organizados por ella el encargo bien determinado de la realización de condiciones efectivas de libertad $^{26} \mathrm{y}$ tal realización también se cumple con el reconocimiento de la participación como una forma expresiva de la dignidad humana, en la medida en que aquella reenvía a la determinación, por parte del ordenamiento, de condiciones que instrumenten la experiencia personal de la autodeterminación y la autorealización.

Precisamente estos recorridos de autorealización y autodeterminación (Selbstverwirklichung und bestimmung) - a los que la Constitución asiste, pero no orienta en su contenido - constituyen uno de los pilares del proceso de integración política, en su dimensión constitucional, en la medida en que permiten la realización de los derechos fundamentales en su historicidad ${ }^{27}$.

La estrecha relación entre dignidad humana y participación en el procedimiento como forma de ejercicio cooperativo de los derechos (con el Estado, o con otros titulares del mismo o de otros derechos fundamentales) contribuye a desvelar la auténtica naturaleza del proceso de integración política como deber histórico de construcción de unidad: en otras palabras, moviéndonos desde una premisa similar, el Estado - y con él, cada proceso de integración de la comunidad política constituido,

\footnotetext{
${ }^{24}$ Garanzie costituzionali..., cit., p. 34 .

${ }^{25}$ Sobre el mismo v. P. RIDOLA, Garanzie costituzionali..., cit., pp. 14 ss.,

${ }^{26}$ En otras palabras, como sugiere H. GOERLICH, Grundrechte..., cit., pp. 190-193, la coordinación estructurada a través de procedimientos - entre libertad negativa y libertad positiva encuentra su expresión constitucional en el reconocimiento de una libertad que se transforma en real en el momento en que se reconoce como igual y fundada sobre caminos de autorealización, articulados también en torno a procedimientos estatales de prestaciones. La libertad - podemos decir con Goerlich, parafraseando a Smend, Heller y el propio Häberle - "reenvía siempre a condiciones establecidas por el derecho, por los derechos subjetivos de los ciudadanos, así como por la exigencia de conformar el poder público a través de la Constitución" (trad. nuestra: cfr. el original "Freiheit besteht immer nur unter Bedingungen des Rechts, subjektiver Rechte der Bürger und der Konstituierung öffentlicher Gewalt durch Verfassung") Sobre el tema ver también E. DENNINGER, Staatliche Hilfe..., cit., pp. 307-309, que enuncia expresamente el ligamen entre protección de la dignidad del hombre y su actuación también a través de instrumentos idóneos para la garantía de la participación en procedimientos.

${ }^{27}$ Cfr. H. GOERLICH, Grundrechte..., cit., p. 347.
} 
organizado, limitado y orientado por el derecho - no representa más una unidad dada, sino que es continuamente re-constituido (integrado) por actos de ejercicio de los derechos fundamentales, de ellos y en ellos es realizado y se renueva, llegando a constituir "el objeto de una construcción comunitaria" ("Gegenstand gemeinschaftlicher Gestaltung",28). Como se ha afirmado, "los particulares llevan a cumplimiento a la capacidad de acción del Estado en el ejercicio y en la realización de los derechos conforme al derecho" 29 , y ello a pesar de la imprescindibilidad del marco constitucional, y por lo tanto de la funcionalidad de la dimensión institucional de los derechos respecto de la protección y la promoción de la dignidad humana, coherentemente con su estructura de validez centrada en la persona humana ("personale Geltungstruktur")

La realización de la libertad exige por lo tanto prestaciones estatales que no se reducen al campo asistencial sino que implican antes de nada una red de condiciones que asistan y acompañen - sobre el plano material, y no sólo - los diferentes caminos de autodeterminación personal, garantizando condiciones de igualdad. En esta óptica "la Constitución crea el marco para las formas y los procedimientos de organización del derecho de prestación, estableciedno una correlación entre el Estado y la sociedad"31. Por esta vía, los derechos fundamentales en el Estado de las prestaciones se convierten «también» en garantías de participación en los procedimientos y puede hablarse de una libertad positiva constitucionalmente orientada (Verfassungsorientierte positive Freiheit), cualificada en sentido cooperativo; aun debe señalarse que es precisamente la referencia a la cooperación en el cuadro constitucional la que aleja los riesgos de una deriva institucional. El ligamen entre dimensión institucional de los derechos fundamentales y la legitimación del sistema constitucional es de este modo ulteriormente aclarado a través de la referencia a las garantías procedimentales como instrumento de participación.

La orientación del Estado prestacional hacia los derechos fundamentales se hace posible, por lo tanto, sólo si se convierte en "Estado constitucional prestacional", es decir, Estado conformado por una Constitución que disciplina y promueve las

\footnotetext{
${ }^{28}$ Cfr. P. HÄBERLE, Grundrechte im Leistungsstaat, cit., p. 58.

${ }^{29}$ Cfr. H. GOERLICH, Grundrechte..., cit., pp. 371-372, (tr. nuestra, cfr. el texto original: «Die einzelnen Menschen vollziehen Hervorbringung und Zuordnung der Handlungskapazitäten eines Staates in Rechtsausübung und Rechtsverwirklichung der Grundrechte»). En este sentido, por otro lado, el Estado no puede comprenderse como un dato a priori, sino como obligación, continuamente realizada en el ejercicio de los derechos fundamentales personales y políticos, y significativamente Goerlich pude concluir afirmando - y se nota la influencia de Heller - que "Die Konstituierung der Wirkungseinheit des Gemeinswesens ist nur auf die Grundrechte als Verfahrensgarantien zurückzuführen" ("la constitución de la unidad real de la comunidad puede reconducirse sólo a los derechos fundamentales como garantías procesales").

${ }^{30}$ H. GOERLICH, Grundrechte..., cit., p. 378.

${ }^{31}$ Cfr. p. 149 de la traducción italiana. En otras palabras, la igualdad, reacciona sobre el sistema de libertad típica del constitucionalismo liberal, estimulando, mediante la constitución, la fundación y la disciplina de instrumentos (también organizativos y procedimentales) destinados a garantizar el efectivo disfrute de los derechos. Esto significa - con Häberle - que el Estado de las prestaciones debe crear premisas y condiciones efectivas para que todos podamos hacer el mismo uso de la libertad constitucionalmente garantizada: en ausencia de tales premisas la libertad que privada de valor ("Freiheit ist ohne die tatsächlichen Voraussetzungen [...] wertlos”, cfr. p. 96 de original en alemán.
} 
condiciones idóneas a la plena realización de los derechos fundamentales, porque "el Estado de las prestaciones en absoluto no puede pensarse sin la realización plena (reale Geltung) de los derechos fundamentales" ${ }^{32}$. En este sentido puede citarse la importante afirmación häberliana según la cual "el Estado de las prestaciones se presenta $[\ldots]$ como Constitución vivida precisamente en sus «funciones de prestación»", en cuyo ámbito "las directrices y los procedimientos conexos a estas funciones «llevan al cumplimiento» del Estado social «desde abajo»"33.

Mediante los derechos fundamentales - conjugados también como garantías procedimentales e instrumentos de participación - se realiza una «praktische Sozialisierung» de los derechos fundamentales, es decir una difusión del gozo efectivo de los derechos en la sociedad mediante su ejercicio cooperativo y "participativo" con la finalidad de favorecer y hacer posible la "realización social de los derechos fundamentales" (soziale Grundrechtserfüllung), entendida como "creación de una efectiva igualdad de oportunidades"34. Sobre esta base se pueden comprender afirmaciones significativas, como aquellas que disponen que la realización de la libertad en la igualdad no es consecuencia de la autoregulación social ("gesellschaftlicher Selbstregulierung") sino que requiere de acciones estatales de dirección y prestación ("leistender und leitender Staatstätigkeit") ${ }^{35}$. Un Estado prestacional orientado a los derechos puede constituir por tanto, según Häberle, una respuesta eficaz al «déficit» de efectividad de los derechos sociales, mediante una configuración del ejercicio cooperativo de los derechos mediante la participación en procedimientos como instrumento de la mediaciòn entre plena realización de la igualdad y salvaguardia de la libertad ${ }^{36}$ : en este sentido, la Constitución "en su vinculación con la realidad social se convierte en ámbito y programa social de una «res publica semper reformanda», es decir sustancia de obligaciones [...] los derechos fundamentales y el Estado de las prestaciones organizado según la Constitución se destinan a la realización del objetivo de hacer posible una vida libre a través de la igualación social correspondiente a cánones de humanidad" ${ }^{\prime 37}$. Sólo si se inscribe el derecho estatal

${ }^{32}$ Cfr. P. HÄBERLE, p. 55 del original en alemán.

${ }^{33}$ ID., p. 136 tr. it.

${ }^{34}$ Tr. nuestra: cfr. El original en alemán, p. 84: “Das Stichwort für „Grundrechte im Leistungsstaat“ lautet daher: „soziale Grundrechtserfüllung“, $d$. h. Schaffung tatsächlicher Chancengleichheit in realer grundrechtlicher Freiheit für Alle (Abbau unzumutbarer witscheftlicher Barrieren), auch um dem Preis der Begrenzung übermäßiger Freiheit weniger" (corsivi aggiunti).

${ }^{35}$ Cfr. p. 97 de original en alemán.

${ }^{36}$ También en este sentido es posible decir, con Ridola, que la dimensión positivo/participativa de la libertad en el Estado de las prestaciones se muestra como "fattore essenziale di trasformazione e di mobilità sociale" (Garanzie costituzionali..., cit., p. 22). Esta consiste en otras palabras en una "domanda attiva, di libertà, indispensabile alla liberazione dalle condizioni sociali di oppressione o di predominio che ostacolano la realizzazione delle sfere della personalità tutelate a livello costituzionale" (p. 23), a partir, por otro lado, de la posibilidad de una "lettura integrata del principio pluralista e del principio di uguaglianza come pilastri della garanzia di una società aperta che rende possibile la realizzazione di identità molteplici". Sobre el tema v. aun, amplius, ID. I diritti fondamentali..., cit., pp. 123 ss., 148 ss.

${ }^{37}$ Tr. nuestra: cfr. el original en alemán en las pp. 98-99: die Verfassung wird zu „wircklichkeitsbezogener Rahmen und Sozialprogramm einer „res publica semper reformanda“, Substanz von Aufgaben. Grundrechte und verfasster Leistungsstaat dienen dem Ziel, freiheitliches Leben in sozialem Ausgleich und Humanität zu ermöglichen“. 
prestacional en el cuadro de una Constitución que ve realizado su propio carácter de proceso abierto también a través de la participación como forma de ejercicio de los derechos, el intento de conciliar libertad e igualdad salvaguarda la dimensión plenamente subjetiva del derecho fundamental, alejando el riesgo de convertirse en un Estado social-asistencial «total» ("Umschlag zum totalen Wohlfahrsstaat"), en el que la relación entre libertad e igualdad sufre una torsión hacia la absolutización de la segunda ${ }^{38}$.

La dimensión procesal, desde este punto de vista, se transforma en uno de los elementos cualificantes que Hesse define - de manera muy eficaz - el "«status» fundamental de derecho constitucional" (Verfassungsrechtlicher Grundstatus) que describe tanto la relación ciudadano y poder público cuanto la trama de la convivencia entre ciudadanos en el Estado constitucional abierto. Un «status» que en Hesse - debe precisarse en seguida - no se limita a describir la articulación formal de tales relaciones, si no que se plantea como «status» de derecho material (materiellrechtlicher Grundstatus), vehículo de contenidos constitucionales en tanto que instrumento de actuación y realización de derechos.

3. Llegamos así al corazón de la construcción häberliana de la relación entre derechos, procedimiento y legitimación del Estado constitucional o, dicho de otro modo, la integración - debatida y no exenta de perfiles problemáticos - de la teoría jellinekiana de los «status» a través del llamado «status activus processualis».

Esta se basa, como precisa el propio autor, en la premisa metodológica de la "liberación de la dogmática" (Befreiung der Dogmatik) ${ }^{39}$ de la observancia ritual de esquemas tradicionales en el marco de una construcciòn de los conceptos jurídicos atenta a su dimensión histórica. Esta última se fundamenta, a su vez, sobre la plena consciencia de la tupida red de interdependencias que ligan la elaboración teóricodogmática de los derechos fundamentales a su efectividad en la experiencia constitucional. Si es cierto que la dogmática tiene el deber de "sostener" los derechos fundamentales en el complejo proceso de evolución y mutación del cuadro histórico y ético-social de referencia, de ello deriva la exigencia de una "apertura" de la propia dogmática, con la finalidad de "hacer sensibles los derechos fundamentales frente a la realidad social". Una correcta diferenciación entre elaboración dogmática y "dogmatismo" puede entonces facilitar el desarrollo del deber propio de la dogmática, que es aquel de dar una "respuesta a desafios concretos", desarrollando "garantías para la racionalidad de la decisión jurisprudencial" ". Junto a la necesidad de una elaboración dogmática articulada de modo cierto y racional ${ }^{41}$, aparece la consciencia

\footnotetext{
${ }^{38}$ Cfr. las pp. 102-103 del original en alemán.

${ }^{39}$ Cfr. p. 81 del original en alemán.

${ }^{40}$ Cfr. le pp. 168-169 de la traducción italiana. Para una reflexión en profundidad sobre la relación entre dogmática y dogmatismo, fundamental A. A. CERVATI, Per uno studio comparativo..., cit., especialmente los cap. I, II, III, VI.

${ }^{41}$ Es el propio Häberle quien afirma que "proprio nell'ambito dei diritti fondamentali è irrinunciabile una dogmatica che tenti di dimostrarsi razionale, che riveli i "veri" motivi e che includa attendibili controlli su se stessa" por que "se così non fosse, l'interpretazione dei diritti fondamentali si trasformerebbe in decisione, la cui conseguenza sarebbe il caos giuridico delle opinioni” (cfr. p. 170 de la trad. it.).
} 
de la exigencia de una continua "revisión de las soluciones de los problemas" que los salve de una dogmatización: en este sentido, la obra del jurista debe ser entendida no (sólo) como conservación de lo existente, sino (también) como conciliación de la instancia de la seguridad jurídica con el avance de la sociedad.

Sobre estas bases, Häberle avanza la propuesta de una integración de la «Statuslehre» de G. Jellinek, cuya impronta "absolutista tardía” debe ser transferida sobre sólidos fundamentos democráticos ${ }^{42}$ a través de la determinación de un nuevo «status» que, sin sustituir a los cuatro determinados por el propio Jellinek, haga posible cualificar la relación entre Estado y ciudadano de forma más adecuada al nuevo contexto histórico, político y constitucional. Si, por un lado, se mantiene la idea de fondo de la elaboración jellinekiana - el concepto de «status», que describe sobre el plano del derecho público la relación entre Estado y ciudadano ${ }^{43}$ - por otro lado la introducción del «status activus processualis» hace visible la nueva articulación de esta relación en el Estado prestacional, describiendo una nueva dimensión coherente con el desarrollo histórico. Desde un ligamen "monodimensional", fundado sobre la dialéctica del derecho fundamental y la intervención limitativa "intrusiva" por parte del Estado, evoluciona a una relación "multilateral" de prestación que despliega sus efectos propios también en el plano de las relaciones sociales "horizontales": es más, es precisamente en este espacio que pueden resolverse las colisiones entre derechos fundamentales. En otras palabras, ésta se convierte en "vehículo de concordancia práctica entre derechos fundamentales y Estado de las prestaciones", en la medida en que el procedimiento garantiza la confrontación entre los intereses en juego antes de que entren en conflicto ${ }^{44}$.

La nueva relación entre ciudadano y estado descrita mediante el «status activus processualis» se expresa principalmente en la dimensión procedimental de las libertades fundamentales ("Er meint die verfahrensrechtliche Seite grundrechtlicher Freiheit"45).

\footnotetext{
${ }^{42}$ Cfr. El original en alemán (p. 80): "sie ist von ihrem spatabsolutistischen Kopf auf demokratische Füße zu stellen".

${ }^{43}$ G. JELLINEK, System der subjektiven öffentlichen Rechte, Tübingen, J. C. B. Mohr 1892, p. 78: status es la cualificación más exacta desde un punto de vista jurídico de la noción misma de personalidad, conjugada en sentido positivista (cfr. el texto alemán: „Die Persönlichkeit ist theoretisch eine das Individuum qualificirende Relation desselben zum Staate. Sie ist juristisch daher ein Zustand, ein Status, an den das einzelne Recht anknüpfen kann, der aber selbst nicht Recht ist“) Derecho público subjetivo son las pretensiones que resultan del status y aparecen conectadas a la multiplicidad de relaciones con el Estado de las cuales el individuo es parte en virtud de su propia "pertenencia" (Zugehörigkeit) al Estado (p. 81) En consecuencia, el concepto de personalidad, o status, describe el conjunto de las diversas facultades - reconocidas y conferidas por el ordenamiento jurídico - que reenvían, en su complejidad, a la posibilidad de activar (,,in Bewegung zu setzen”) al Estado, es decir, las normas de su ordenamiento en interés individual (cfr. p. 53).

${ }^{44}$ Tr. nuestra: cfr. p. 87 del original en alemán: „Das eindimensionale Verhältnis Individuum-Staat (Grundrecht-Eingriff) wird durch eine Vermehrung des Kreises der von Staatsleistungen in Grundrechtinteressen betroffenen einzelnen und Gruppen zu einem „mehrseitigen“ Leistungsverhältnis, das kollektive Wirkungen intendiert; in seinem Rahmen sind Grundrechtskollisionen zu lösen [...] Das vorgezogene - Verfahren kann einen Ausgleich der einander widerstreitenden Gesichtspunkte gewährleistenden, bevor es zum Konflikt kommt; es wird zum Vehikel „praktischer Konkordanz“ (Hesse) zwischen Grundrechten und Leistungsstaat".

${ }^{45}$ Cfr. p. 51 del original en alemán.
} 
La superación de la centralidad del «status negativus» (y bien mirado del propio «status passivus») se abre a una plena realización del «status activus» en el cuadro del ejercicio responsable y participativo de los derechos como canal de afirmación de una ciudadanía conjugada en el sentido de la «Mitgliedschaft» (membership, condición de miembro) más que en sentido de la mera «Zugehörigkeit» (pertenencia) al Estado. Al mismo tiempo, la reafirmación del momento participativo permite encajar la organización de la libertad, como se ha visto, con la protección de la dignidad de la persona humana en sus concretas manifestaciones históricas. La novedad en la relación de «status» introducida por Häberle reside por lo tanto en la centralidad de la autodeterminación, profundamente condicionada por la Constitución como pilar de una nueva relación entre público y privado, orientada a la construcción de procesos de integración. Esta, por lo tanto, no implica más la posibilidad única de accionar normas del ordenamiento jurídico del Estado en defensa del propio interés ${ }^{46}$, sino que se abre a la posibilidad de autodeterminarse a través del ejercicio de los propios derechos mediante los procedimientos de prestación; que, a su vez, estructuran el vínculo entre el ciudadano y el Estado en torno a la cooperación en la determinación del contenido específico del derecho interesado por la actividad estatal de decisión y prestación. La consecuencia, como se ha dicho más veces, es la de una verdadera y propia apertura de las funciones de prestación - y del aparato organizativo llamado a ejercerlas —al ciudadano y a los sujetos del pluralismo social ${ }^{47}$.

Sobre la base de cuanto se ha afirmado a propósito de la fundación dogmática del «status activus processualis», se puede entender completamente la afirmación de Häberle cuando dispone que desde estas premisas la dogmática de los derechos fundamentales puede (y debe) elaborar un instrumentario polivalente, en más estratos, (mehrwertiges und mehrschichtiges Instrumentarium) que conciba las garantías de los derechos fundamentales sociales en el sentido más amplio, como objetivo de la Constitución y mandatos de tutela social gradualmente estructurados, como derechos subjetivos de prestación y principios de interpretación para la jurisprudencia" (172).

En otras palabras, no es posible limitar el alcance del «status activus processualis» a la introducción de nuevas figuras de derechos fundamentales "al” o

\footnotetext{
${ }^{46}$ En el pensameinto de Jellinek, por otro lado, tal posibilidad no sólo resumía - como se ha visto - el núcleo lógico del concepto de status, sino que describía la deiferencia entre derechos ubjetivos y derechos reflejos (cfr. G. JELLINEK, System..., cit., p. 66 ss.). Si continúa siendo verdad, en línea general, que el derecho público subjetivo del súbdito (Subjicirten) no sólo es creado y protegido, sino también realizado (realisirt) por el derecho objetivo (p. 66), el criterio formal de distinción entre las dis figuras se encuentra en la circunstancia de que el derecho público subjetivo, en tanto que expresión de un status que cualifica la realización entre individuo y Estado, confiere al particular la capacidad de accionar en su propio interés normas del ordenamiento jurídico: y ello porque la existencia de derechos públicos subjetivos se justifica en "la consideración de orden general según la cual no todas las proposiciones jurídicas son dictadas en interés general [...] sino que existe un amplio ámbito de normas jurídicas, que tiene la finalidad predominante de servir a intereses individuales” (tr. nuestra: cfr. el original en alemán, p. 74 :,,aus dem allgemeinen Gesichtspunkte, dass nicht alle Rechtssätze ausschliesslich im Gemeininteresse gegeben sind [...] es giebt vielmehr ein sehr ausgedehntes Gebiet von Rechtsnormen, die den überwiegendem Zweck haben, individuellen Interessen zu dienen“").

${ }^{47}$ Cfr. p. 90 de original en alemán: "dieser status activus processualis öffnet auf verfahrensmäßige Weise die Leistungsfunktionen zum Bürger und zu den Pluralgruppen hin“, pero también supra, nota 16.
} 
"en el" procedimiento ni conjugar las garantías procesales de los derechos en sentido exclusivamente instrumental a la determinación de remedios jurisdiccionales eficaces ${ }^{48}$. Al mismo tiempo, la elaboración teórica de Häberle y en particular el vínculo entre dimensión material y dimensión procesal de los derechos fundamentales - que se manifiesta en la influencia directa del titular, a través de la participación, sobre la determinación del contenido del derecho en sede del procedimiento público de decisión - muestra que el interés en el procedimiento, lejos de sustituirse en interés del resultado, se integra con él ${ }^{49}$.

El "descubrimiento" del «status activus processualis» como nueva manifestación de la relación entre ciudadano y Estado, entre esfera pública y esfera privada irradia en una suerte de «Ausstrahlungswirkung» orientada al procedimiento- sobre el contenido del derecho ya sea como integración de la facultad de disposición, sea como forma de participación en la determinación del contenido, contribuyendo a diseñar la figura del "nuevo ciudadano" como sujeto empeñado responsablemente a dotar de sentido la propia pertenencia a la comunidad estatal ${ }^{50}$. La citada «Mitgliedschaft» (condición de miembro), dicho de otro modo, no se basa sólamente en la «gliedliche Stellung» del particular en el Estado ${ }^{51}$ - sobre la idea de que la condición de miembro descienda de un posicionamiento del poder público, manifestada mediante el reconocimiento normativo de espacios de libertad - sino que se abre a una

\footnotetext{
${ }^{48}$ En este sentido, vg., R. ALEXY, Teoría de los derechos fundamentales, Madrid, CEC, 1993, pp. 457-458, así como pp. 472 ss. En el Estado prestacional, por el contrario, los derechos fundamentales articulan la propia función de "normas de estructura" no sólo dentro de los tribunales, sino también fuera de ellos, o mejor aun: antes del proceso ("vorgerichtlich", cfr. P. HÄBERLE, p. 129 del original en alemán)

${ }^{49}$ En el sentido, sin embargo en que "Las exigencias jusfundamentales al procedimiento no pueden substituir las exigencias fundamentales al resultado", cfr. R. ALEXY, op. cit., p. 473. Más cercano a lo que se sostiene en el texto, E. DENNINGER, Staatliche Hilfe..., cit., p. 308 que afirma que el resultado podrá también ser contrario en todo o en parte al interesado, pero habrá debido tener también una posibilidad adecuada ("eine faire Chance") de influir en él.

${ }^{50}$ La expresión "nuovo cittadino" deriva del importante ensayo de Feliciano BENVENUTI (Venezia, Marsilio 1994, del cual se señalan en particualr las pp. 60 ss. sobre la relación entre participación y libertad positiva, y las pp. 104 ss. Para la importante señalación de la relación entre responsabilidad "participativa" del ciudadano y procesos educativos centrados sobre las libertades culturales), y se reenvía a itinerarios fecundos de la reflexión administrativista italiana de los últimos decenios, que han individuado en el procedimiento no sólo un instituto "servente" respecto al principio de legalidad - en el sentido de la conjugación del principio del justo procediemiento ofrecida por Vezio CRISAFULLI comentando la sentencia n. 13/1962 de la Corte costituzionale (Principio di legalità e "giusto procedimento", in Giur. cost. 1962, pp. 130 ss.) - sino también, de forma más general, un instrumento de apertura de la actividad administrativa en la participación democrática (o "demarchica", por usar un término propio de Benvenuti) de los ciudadanos sobre el fondo de una revisitación radical de la relación entre privado y público, precisamente en el sentido de la apertura, de la comunicací́n y de la cooperación: pensemos, por ejemplo, en la obra de Giorgio BERTI, y en particular en Procedimento, procedura, partecipazione, en Studi in memoria di E. Guicciardi, Pádua, CEDAM, 1975, pp. 779 ss., especialmente pp. 788 ss. Más recientemente , v. G. COLAVITTI, Il "giusto procedimento" come principio di rango costituzionale, en http://www.associazionedeicostituzionalisti.it/dibattiti/amministrazione/colavitti.html. El propio P. HÄBERLE, subraya el estrecho vínculo entre la propia propuesta respecto de la concepción de los Grundrechte y una figura del ciudadano informada, comprometida y responsable, cuya "simbiosis" con la administración determina nuevas formas de colaboración con los grupos y la administración misma, en el sentido del status activus processualis (cfr. pp. 88-89, también 127 del original en alemán).

${ }^{51}$ Como quería G. JELLINEK, System..., cit., p. 50.
} 
continua redefinición mediante el ejercicio de los derechos en la evolución histórica de la comunidad.

El vínculo entre dimensión procesal y dimensión material del derecho fundamental - o, parafraseando a Häberle, el "refuerzo" de la dimensión material "mediante" el procedimiento $^{52}$ - resulta, en este punto, de una pluralidad de factores y se desarrolla sobre más planos: en primer lugar, la dimensión procesal constituye un componente autónomo del contenido del derecho, en la medida en que se traduce en facultad de participación en los procedimientos de decisión pública relativos al derecho fundamental concreto. En segundo lugar, a través del ejercicio de dichas facultades, el titular participa en la determinación del contenido concreto del derecho fundamental.

\section{BIBLIOGRAFIA}

ALEXY, R., Teoría de los derechos fundamentales, Madrid, CEC, 1993.

AZPITARTE SÁNCHEZ, M., Apuntes sobre el pensamiento de Peter Häberle en el contexto de la dogmática alemana, en Rev. Fac. Der. Univ. Granada n. 6/2003, pp. 345 ss.

BENVENUTI, F., Il nuovo cittadino, Venezia, Marsilio 1994.

BERTI, G., Procedimento, procedura, partecipazione, en Studi in memoria di E. Guicciardi, Pádua, CEDAM, 1975.

CERVATI, A. A., Il diritto costituzionale e la comparazione nel pensiero di Peter Häberle, en ID. Per uno studio comparativo del diritto costituzionale, Turín, Giappichelli 2009, pp. 140 ss.

COLAVITTI, G., Il "giusto procedimento" come principio di rango costituzionale, en http://www.associazionedeicostituzionalisti.it/dibattiti/amministrazione/colavitti.html

CRISAFULLI, V., Principio di legalità e "giusto procedimento”, in Giur. cost. 1962, pp. 130 ss.

DENNINGER, E., Staatliche Hilfe zur Grundrechtsausübung durch Verfahren, Organisation und Finanzierung, en J. ISENSEE- P. KIRCHHOF (hrsg.), Handbuch des Staatsrechts, Heidelberg, C. Müller, 1992.

GOERLICH, H., Grundrechte als Verfahrensgarantien, Baden Baden, Nomos 1981.

HÄBERLE, P., Grundrechte im Leistungsstaat, en VER. DER DEUTSCHEN STAATSRECHTSLEHRER, Grundrechte im Leistungsstaat/Die Dogmatik der Verwaltungsrechts vor den Gegenwarts Aufgaben der Verwaltung, Berlin-New York, De Gruyter 1972, pp. 43 ss., trad. it. I diritti fondamentali nello Stato delle prestazioni, en P. HÄBERLE, Cultura dei diritti e diritti della cultura nello spazio costituzionale europeo, Milán, Giuffré, 2003, pp. 133 ss.

HELlER, H., Democrazia politica ed omogeneità sociale (1928), en Stato di diritto o dittatura?, U. Pomarici (ed.), Nápoles, ESI, 1998, pp. 9 ss.

HESSE, K., Grundzüge des Verfassungsrechts der Bundesrepublik Deutschland, 20. Aufl., Heidelberg, C. F. Müller 1999.

HESSE, K., Bedeutung der Grundrechte, en BENDA/MAIHOFER/VOGEL, Handbuch des Verfassungsrechts der Bundesrepublik Deutschland, Berlin, De Gruyter 1994, pp. 127 ss.

HESSE, K., Bestand und Bedeutung der Grundrechte in der Bundesrepublik Deutschland, in EuGrZ 1978, pp. 427 ss.

JELLINEK, G., System der subjektiven öffentlichen Rechte, Tübingen, J. C. B. Mohr 1892.

${ }^{52}$ Cfr. El original en alemán: "über Verfahren wird die materiellrechtliche Seite der Grundrechte im Leistungsstaat gestärkt" (p. 86). 
RIDOLA, P., Diritti fondamentali. Un'introduzione, Turín, Giappichelli 2006.

RIDOLA, P., Democrazia pluralistica e libertà associative, Milán, Giuffré 1987.

RIDOLA, P., Garanzie costituzionali e dimensioni dei diritti di libertà, en ID. Diritti di libertà e costituzionalismo, Turín, Giappichelli 1997.

RIDOLA, P., Laudatio de Peter Häberle, en Rev. Der. Const. Eur., n. 1/2009, pp. 389 ss.

RIDOLA, P., Prime riflessioni sullo "spazio pubblico" nelle democrazie pluralistiche, en prensa en curso de publicación en el Liber amicorum per Federico Spantigati y en P. RidolA, Diritto comparato e diritto costituzionale europeo, Torino, Giappichelli, 2010.

STERN, K., Das Staatsrecht der Bundesrepublik Deutschland, Bd. III/1. Allgemeine Lehren der Grundrechte, München, Beck, 1988. 